\title{
Management of MANEC of the colon and rectum: A comprehensive review of the literature
}

\author{
ANNA PASPALA $^{1}$, NIKOLAOS MACHAIRAS ${ }^{1}$, ANASTASIA PRODROMIDOU $^{1}$, \\ ELEFTHERIOS SPARTALIS $^{1}$, ARGYRIOS IOANNIDIS ${ }^{2}$, IOANNIS D. KOSTAKIS ${ }^{1}$, \\ DIMETRIOS PAPACONSTANTINOU $^{1}$ and NIKOLAOS NIKITEAS ${ }^{1}$
}

${ }^{1}$ Laboratory of Experimental Surgery and Surgical Research, Medical School, National and Kapodistrian University of Athens, 11527 Athens; ${ }^{2}$ Second Department of General Surgery, 'Sismanoglio' General Hospital, 15126 Athens, Greece

Received May 7, 2018; Accepted June 8, 2018

DOI: $10.3892 / \mathrm{mco} .2018 .1649$

\begin{abstract}
Mixed adeno-neuroendocrine carcinoma (MANEC) is a rare pathological diagnosis recently acknowledged by the World Health Organization in 2010. MANEC is a neoplasm characterized by significant histological heterogeneity and is characterized by the simultaneous presence of both adenocarcinomatous and neuroendocrine differentiation; their definition includes each component found in at least $30 \%$ of the tumor. Colorectal MANEC constitutes an uncommon type of malignant tumor. The true prevalence of colorectal MANEC has not been precisely defined and published studies are limited to case reports and small case series. The aim of the present review was to accumulate the existing evidence on colorectal MANEC with special attention to the clinicopathological characteristics, management and survival rates of patients diagnosed with this malignancy. A total of 20 studies (16 case reports and 4 retrospective cohorts) reported outcomes for patients with colorectal MANEC and were finally considered eligible for analysis. The results of the present study show that patients with early stage MANEC have more favorable survival compared to those diagnosed in advanced stages. Due to its neuroendocrine nature, which is characterized by rapid progression, MANEC is diagnosed in advanced stages in the majority of cases and thus potentially explains the poor survival rates. Because of its aggressive nature and high recurrence rate, adjuvant chemotherapy constitutes a critical part of the treatment and significantly improves survival. Further larger studies are needed in order to establish guidelines for the treatment of these rare lesions.
\end{abstract}

Correspondence to: Dr Nikolaos Machairas, Laboratory of Experimental Surgery and Surgical Research, Medical School, National and Kapodistrian University of Athens, 15772 Athens, Greece E-mail: nmachair@gmail.com

Key words: mixed adeno-neuroendocrine carcinoma, colorectal, chemotherapy

\section{Introduction}

NEC of the colon and rectum is a rare entity and accounts for less than $2.0 \%$ of all colorectal malignancies (1). Mixed adeno-neuroendocrine carcinoma (MANEC) is a rare pathological diagnosis recently acknowledged by the World Health Organization in $2010(2,3)$.

MANEC is a neoplasm characterized by significant histological heterogeneity and is distinguished by the simultaneous presence of both adenocarcinomatous and neuroendocrine differentiation; their definition includes each component found in $\geq 30 \%$ of the tumor (3). In addition, 2 of 3 commonly used immunohistochemical neuroendocrine markers, chromogranin A (CgA), synaptophysin (Syn) and CD56, should be positive to substantiate the neuroendocrine differentiation of MANEC (4). In the case of colorectal MANEC, the currently available data derive from case reports and small case series and therefore the clinical behavior, the optimal management and the prognosis of colorectal MANEC remain to be determined.

The aim of the present review was to accumulate the existing evidence on colorectal MANEC with special attention to the clinicopathological characteristics, management and survival rates of patients diagnosed with this malignancy.

\section{Materials and methods}

Study design. All appropriate observational studies and case reports addressing cases of patients who were diagnosed with a tumor characterized as colorectal MANEC were considered eligible for inclusion in the present systematic review. Reviews and animal studies were excluded from analysis and tabulation. No language restrictions were performed. NM and AP independently performed a meticulous search of the literature, excluded overlaps, and tabulated the selected indices in structured forms. Articles, which did not report data concerning clinical characteristics of the included patients, were excluded. Consensus of all authors resolved potential discordances in methodology, selection of articles, and statistical analysis.

Search strategy and data collection. We systematically searched for articles published up to March 2018 using 
Medline (1966-2018), Scopus (2004-2018), and Google Scholar (2004-2018) databases along with the references of the articles, which were retrieved in full text. The following key words were used for the search: 'colorectal', 'gastrointestinal tract', 'MANEC', and 'mixed adenoneuroendocrine carcinoma'. A minimum number of search keywords were utilized in an attempt to assess an eligible number that could be easily searched while simultaneously minimizing the potential loss of articles. Articles that fulfilled or were deemed to fulfil the inclusion criteria were retrieved.

Data on patient characteristics included age and sex, while disease characteristics included tumor site, type of surgery and adjuvant surgery along with histopathological and immunochemical findings of the tumor. Concerning the main findings of the study, follow-up period, survival rates and mortality were evaluated.

\section{Results}

Included studies. A total of 18 studies (14 case reports and 4 retrospective cohorts) reported outcomes for patients with colorectal MANEC and were finally considered eligible for analysis (5-22). Data on 93 patients (62 male and 31 female; age range of 32-96 years) was retrieved from the selected studies. The analysed indices are shown in Table I and included cumulative outcomes of the total of the included patients. In $69 \%$ $(n=9$ out of 13) of cases, patients presented with pain and signs of obstruction (nausea, vomit and abdominal bloating) (7). Three patients were asymptomatic while 3 had anaemia as the primary finding. MANEC of the right colon was the histological diagnosis in 29 cases, 25 cases had left colon disease while the lesion was located in the transverse colon in 10 cases and in rectum in 12 . Additionally, one study reported 17 patients with colon MANEC but the exact colon site was not reported. Lymph node metastasis was detected in 65/90 (72.2\%) patients whereas 18/90 (20\%) patients presented with distant metastasis. The liver was the most common metastatic site followed by bone metastasis. A total of 25 patients received postoperative adjuvant chemotherapy. Immunochistochemical analysis revealed CgA-positive tumors in $52.4 \%$ ( $\mathrm{n}=43$ out of 82 ) of patients and Syn-positive tumors in $89 \%$ ( $n=74$ out of 83 ) of cases. A total of 16 studies reported outcomes with regard to adjuvant chemotherapy administration, with $25 / 61$ patients receiving adjuvant chemotherapy.

Main outcomes. La Rossa et al reported one postoperative death, $9(59 \%)$ patients succumbing to the disease and 3 (25\%) patients alive without recurrence during the follow-up period (14). The same study detected no difference in survival rates among patients with NEC and MANEC $(\mathrm{P}=0.82)(14)$. By contrast, in the study by Watanabe et al both 5 -year disease free survival (5-DFS) and 5-year overall survival (5-OS) were significantly lower in the MANEC cases when compared to those diagnosed with adenocarcinoma (60.5 vs. $76.2 \%$, $\mathrm{P}=0.032$ and 69 vs. $82 \%, \mathrm{P}=0.048$, respectively) (5). Those authors presented a 5-DFS of $100 \%$ in patients with stage I tumors, whereas patients with stage II and III were reported with a relatively lower 5-DFS of 72.7 and $47.1 \%$, respectively (5). Komatsubara et al reported $3 / 6$ patients without recurrence during a median follow up of 127 months while
3 succumbed to the disease after a median of 31 months after diagnosis (8). Furthermore, cumulative data from the 14 case reports revealed a survival of $63.6 \%(n=7$ out of 11$)$ in a median follow-up of 24 months, and $36.4 \%$ ( $n=4$ out of 11) patients who died in a median follow-up of 5.5 months. Of note, none of the alive patients were diagnosed with recurrent disease. From the patients who died, all were diagnosed with liver recurrence and/or progression of their liver disease except one who died in the postoperative period (13).

\section{Discussion}

Colorectal MANEC is an uncommon type of tumor. The true prevalence of MANEC has not been precisely defined and published studies are limited to case reports, and small case series. As a result, further research and evaluation is precluded due to their entity. Recently, the largest retrospective case-matched study on patients with MANEC by Watanabe et al, reported a prevalence of $3.2 \%$ of MANEC from the total of patients with colorectal diseases as derived from the hospital records (5). Due to its dual histological profile, both clinical behavior and management of MANEC were substantially different from those of adenocarcinoma and primarily influenced by the contribution of each component as well as the type of cells in the neuroendocrine component. La Rossa et al reported that patients with MANEC, which was composed of large neuroendocrine cells, have a better survival and clinical behavior compared to patients with non-large neuroendocrine cells (small-to-intermediate or mixed large-to-intermediate cell) (14).

Specific clinical practice guidelines have not yet been developed and the latest version of National Comprehensive Cancer Network Guidelines (version 1.0 2017) did not enroll the management of MANEC (23). Contrary to NEC, MANEC requires more complex management, including administration of a patient-tailored chemotherapy according to the histology of their tumor. The only therapeutic strategy for MANEC is complete surgical resection (R0 resection) for both primary tumor and metastases (23). Because of its aggressive nature and high recurrence rate, adjuvant chemotherapy constitutes a critical part of the treatment and significantly improves survival. MANEC comprises two distinctly different components (adenocarcinomatous and neuroendocrine) that have divergent responses to chemotherapy. As a result the most burdensome and challenging part of MANEC patients management is the choice of the suitable chemotherapy for both primary tumor and distant metastases. According to the European Neuroendocrine Tumor Society guidelines, cisplatin and etoposide are considered to be effective for treatment of gastrointestinal poorly differentiated neuroendocrine tumor (NEC) (5). Additionally, the National Comprehesive Cancer Network suggested carboplatin and etoposide or cisplatin and irinotecan as an optimal adjuvant chemotherapy (24). In the present study, 25 from a total of 61 patients received postoperative adjuvant chemotherapy. Seven patients received Folfox, 4 Folfiri, 4 patients received 5-FU, one cisplatinum and etoposide, 2 patients received IRIS, two XELOX and Bevasizumab, 2 patients XELOX and 3 patients received cetuximab and Folfox. A total of 6 out of 26 patients who received adjuvant chemotherapy died. 
Table I. Study characteristics.

\begin{tabular}{|c|c|c|c|c|c|c|c|c|c|}
\hline Authors, year & Patients & $\begin{array}{c}\text { Sex } \\
\text { (female) }\end{array}$ & $\begin{array}{l}\text { Age, years } \\
\text { (median) }\end{array}$ & Location & $\mathrm{CgA}$ & $\begin{array}{l}\text { djuva } \\
\text { Syn }\end{array}$ & chemotherapy & Mortality & (Refs.) \\
\hline Jesinghaus et al, 2017 & 19 & 8 & 64.3 mean & $\begin{array}{l}\mathrm{C}=7 \\
\mathrm{R}=2\end{array}$ & 13 & 19 & $\mathrm{Na}$ & N/A & (18) \\
\hline Watanabe et al, 2016 & 42 & 13 & 72 & $\begin{array}{l}\mathrm{RC}=9 \\
\mathrm{TC}=6 \\
\mathrm{LC}=18 \\
\mathrm{R}=9\end{array}$ & 18 & 34 & 15 & N/A & (5) \\
\hline Komatsubara et al, 2015 & 6 & 2 & 65.7 mean & $\begin{array}{l}\mathrm{RC}=3 \\
\mathrm{TC}=1 \\
\mathrm{LC}=1 \\
\mathrm{R}=1\end{array}$ & $\mathrm{Na}$ & $\mathrm{Na}$ & 5 & $3 / 6$ & (8) \\
\hline La Rossa et al, 2012 & 12 & 4 & 72 & $\begin{array}{l}\mathrm{RC}=8 \\
\mathrm{LC}=4\end{array}$ & 6 & 12 & $\mathrm{Na}$ & $10 / 12$ & (14) \\
\hline $\begin{array}{l}\text { Cases/reports } \\
\text { (cumulative outcomes) }\end{array}$ & 14 & 4 & 65.5 & $\begin{array}{l}\mathrm{RC}=9 \\
\mathrm{TC}=3 \\
\mathrm{LC}=2 \\
\mathrm{R}=0\end{array}$ & 6 & 9 & 6 & $4 / 11$ & $\begin{array}{c}(6,7,9-13) \\
(15-17) \\
(19-22)\end{array}$ \\
\hline
\end{tabular}

CgA, chromogranin A; Syn, synaptophysin; RC, right colon; LC, left colon; TC, transverse colon; R, rectum; N/A, not available.

Two minimal immunohistochemical neuroendocrine markers have been recommended by the European Society of Neuroendocrine Tumors guidelines; $\mathrm{CgA}$ and Syn (4). The diagnosis and differentiation of neuroendocrine tumors is based on recognition of immunoreactivity of at least two markers (CgA and Syn) (4). In the present review immunochistochemical analysis revealed $\mathrm{CgA}$ positive tumor in $52.4 \%$ $(n=43$ out of 82$)$ of patients and Syn positive tumor in $89 \%$ $(n=74$ out of 83$)$ of cases.

Compared to survival rates of adenocarcinoma patients, the survival rate of patients with MANEC is substantially worse, mainly due to the high-grade components of neuroendocrine tumors; in the study by Watanabe et al 5-OS and 5-DFS rates were found decreased in patients with MANEC when compared to those of patients with adenocarcinoma $(\mathrm{P}=0.032$ and $\mathrm{P}=0.048$, respectively) (5). Interestingly, the patients enrolled in this study were matched for main characteristics such as age, sex and tumor characteristics (size, site and TNM) (5). On the contrary, when La Rossa et al compared survival rates of patients with NEC and MANEC, no difference was detected $(\mathrm{P}=0.82)$, indicating the potential role of the neuroendocrine component in the prognosis of the disease (14). No specific prognostic factors or parameters are reported in the literature concerning the survival of MANEC patients. La Rossa et al reported some very interesting results about the presence of predicting histological factors which statistically influencing survival rate (14). They reported that vascular invasion and CD117 expression are independent prognostic factors correlating with shorter survival in both NEC and MANEC patient (14).

According to the current literature, this is the first study, which presents a cumulative report of characteristics, management and postoperative outcomes of patients with MANEC. A thorough search of the literature along with the fact that no date or language restrictions were imposed, eliminated the risk of potential loss of articles. Nonetheless, the fact that the majority of the included studies was restricted in case reports and small case series precluded further analysis. Additionally, the retrospective nature of the included observational studies constitutes further potential weaknesses. Finally, the significant heterogeneity of the included studies along with the fact that some parameters were omitted by some studies was another limitation and precluded reaching to firm results; survival outcomes and with regard to overall survival and disease free survival were underreported by the included studies and the exact criteria of the type of chemotherapy were also not precisely defined.

The results of the present study show that patients with early stage MANEC have more favorable survival compared to those diagnosed in advanced stages. Nonetheless, due to its neuroendocrine nature, which is characterized by rapid progression, MANEC is diagnosed in advanced stages in the majority of cases and thus potentially explains the poor survival rates. Further larger studies, which will evaluate the clinical behavior, prognostic factors, survival rates and benefit of systemic chemotherapeutic regimens in patients with colorectal MANEC are imperative in order to provide with guidelines for the treatment of these heterogeneous lesions.

\section{Acknowledgements}

Not applicable.

\section{Funding}

No funding was received. 


\section{Availability of data and materials}

Not applicable.

\section{Authors' contributions}

APa and NM had the principle idea. APr and NM performed the systematic review. APr and DP analyzed and interpreted the data. NM, APr, ES, AI and IDK wrote the manuscript. NN critically revised the manuscript. All the authors have read and approved the final version of this manuscript.

\section{Ethics approval and consent to participate}

Not applicable.

\section{Consent for publication}

Not applicable.

\section{Competing interests}

The authors declare that they have no competing interests to disclose.

\section{References}

1. Staren ED, Gould VE, Warren WH, Wool NL, Bines S, Baker J, Bonomi P, Roseman DL and Economou SG: Neuroendocrine carcinomas of the colon and rectum: A clinicopathologic evaluation. Surgery 104: 1080-1089, 1988.

2. Komminoth P, Arnold R, Capella C, Klimstra D, Klöppel G, Solcia E and Rindi G: Neuroendocrine neoplasms of the appendix. WHO classification of tumours of the digestive system: 126-128, 2010.

3. Bosman FT, Carneiro F, Hruban RH and Theise ND: WHO Classification of Tumours of the Digestive System. 4th edition. World Health Organization, Geneva, 2010.

4. Rindi G, Bordi C, La Rosa S, Solcia E and Delle Fave G; Gruppo Italiano Patologi Apparato Digerente (GIPAD); Società Italiana di Anatomia Patologica e Citopatologia Diagnostica/International Academy of Pathology, Italian division (SIAPEC/IAP): Gastroenteropancreatic (neuro)endocrine neoplasms: the histology report. Dig Liver Dis 43: S356-S360, 2011.

5. Watanabe J, Suwa Y, Ota M, Ishibe A, Masui H, Nagahori K, Tsuura Y and Endo I: Clinicopathological and Prognostic Evaluations of Mixed Adenoneuroendocrine Carcinoma of the Colon and Rectum: A Case-Matched Study. Dis Colon Rectum 59: 1160-1167, 2016.

6. Jain A, Singla S, Jagdeesh KS and Vishnumurthy HY: Mixed adenoneuroendocrine carcinoma of cecum: A rare entity. J Clin Imaging Sci 3: 10, 2013.

7. Morais M, Pinho AC, Marques A, Lopes J, Duarte A, da Silva PC, Lopes JM and Maia JC: Mixed Adenoneuroendocrine Carcinoma Causing Colonic Intussusception. Case Rep Surg 2016: 7684364 , 2016.

8. Komatsubara T, Koinuma K, Miyakura Y, Horie H, Morimoto M, Ito H, Lefor AK, Sata N and Fukushima N: Endocrine cell carcinomas of the colon and rectum: A clinicopathological evaluation. Clin J Gastroenterol 9: 1-6, 2016.

9. Gurzu S, Kadar Z, Bara T, Bara T Jr, Tamasi A, Azamfirei L and Jung I: Mixed adenoneuroendocrine carcinoma of gastrointestinal tract: Report of two cases. World J Gastroenterol 21 : 1329-1333, 2015.
10. Vanacker L, Smeets D, Hoorens A, Teugels E, Algaba R, Dehou MF, De Becker A, Lambrechts D and De Greve J: Mixed adenoneuroendocrine carcinoma of the colon: Molecular pathogenesis and treatment. Anticancer Res 34: 5517-5521, 2014.

11. Yamauchi H, Sakurai S, Tsukagoshi R, Suzuki M, Tabe Y, Fukasawa T, Kiriyama S, Fukuchi M, Naitoh $\mathrm{H}$ and Kuwano H: A case of very well-differentiated adenocarcinoma with carcinoid tumor in the ascending colon. Int Surg 99: 132-136, 2014.

12. Ito $H$, Kudo A, Matsumura S, Ban D, Irie T, Ochiai $T$, Nakamura N, Tanaka S and Tanabe M: Mixed adenoneuroendocrine carcinoma of the colon progressed rapidly after hepatic rupture: Report of a case. Int Surg 99: 40-44, 2014.

13. Marando A, Dainese E, La Rosa S and Capella C: Images in endocrine pathology: Oncocytic differentiation in a mixed adenoneuroendocrine carcinoma of the colon. Endocr Pathol 24: 54-56, 2013.

14. La Rosa S, Marando A, Furlan D, Sahnane N and Capella C: Colorectal poorly differentiated neuroendocrine carcinomas and mixed adenoneuroendocrine carcinomas: Insights into the diagnostic immunophenotype, assessment of methylation profile, and search for prognostic markers. Am J Surg Pathol 36: 601-611, 2012.

15. Minaya-Bravo AM, Garcia Mahillo JC, Mendoza Moreno F, Noguelares Fraguas F and Granell J: Large cell neuroendocrine-adenocarcinona mixed tumour of colon: Collision tumour with peculiar behaviour. What do we know about these tumours? Ann Med Surg (Lond) 4: 399-403, 2015.

16. Koletsa T, Beretouli E, Tziola T, Mavropoulou S and Karayannopoulou G: Neuroendocrine carcinoma in adenoma of the sigmoid. Hippokratia 18: 362-363, 2014.

17. Jiao YF, Nakamura S, Arai T, Sugai T, Uesugi N, Habano W, Suzuki M, Tazawa H and Goukon Y: Adenoma, adenocarcinoma and mixed carcinoid-adenocarcinoma arising in a small lesion of the colon. Pathol Int 53: 457-462, 2003.

18. Jesinghaus M, Konukiewitz B, Keller G, Kloor M, Steiger K, Reiche M, Penzel R, Endris V, Arsenic R, Hermann G, et al: Colorectal mixed adenoneuroendocrine carcinomas and neuroendocrine carcinomas are genetically closely related to colorectal adenocarcinomas. Mod Pathol 30: 610-619, 2017.

19. Cherbanyk F, Gassend JL, Dimitrief M, Andrejevic-Blant S, Martinet O and Pezzetta E: A rare type of colorectal cancer: mixed adeno-neuroendocrine carcinoma (MANEC). Chirurgia (Bucur) 112: 152-156, 2017.

20. Shin SH, Kim SH, Jung SH, Jang JW, Kang MS, Kim SI, Kim JH and Lee JH: High-Grade Mixed adenoneuroendocrine carcinoma in the cecum: A case report. Ann Coloproctol 33: 39-42, 2017.

21. Silva JRD, Pinho RT and Furtado A: A case of a mixed adenoneuroendocrine tumor of the colon. Rev Esp Enferm Dig 109: $673,2017$.

22. Liu XJ, Feng JS, Xiang WY, Kong B, Wang LM, Zeng JC and Liang YF: Clinicopathological features of an ascending colon mixed adenoneuroendocrine carcinoma with clinical serosal invasion. Int J Clin Exp Pathol 7: 6395-6398, 2014.

23. Tanaka T, Kaneko M, Nozawa H, Emoto S, Murono K, Otani K, Sasaki K, Nishikawa T, Kiyomatsu T, Hata K, et al: Diagnosis, assessment, and therapeutic strategy for colorectal mixed adenoneuroendocrine carcinoma. Neuroendocrinology 105: 426-434, 2017.

24. Eriksson B, Annibale B, Bajetta E, Mitry E, Pavel M, Platania M, Salazar R and Plöckinger U; Mallorca Consensus Conference participants; European Neuroendocrine Tumor Society: ENETS consensus guidelines for the standards of care in neuroendocrine tumors: Chemotherapy in patients with neuroendocrine tumors. Neuroendocrinology 90: 214-219, 2009. 